\title{
Transmission Control of Cross-Regional Heterogeneous Networks for Direct Position Determination
}

\author{
Liang Zong $\mathbb{D}^{1},{ }^{1}$ Han Wang $\mathbb{D}^{2,3,4}$ Liangpeng Lu $\mathbb{D}^{2},{ }^{2}$ Yong Bai $\mathbb{D},{ }^{3}$ Chenglin Zhao, \\ Gaofeng Luo, ${ }^{1}$ and Feng Lin ${ }^{1}$ \\ ${ }^{1}$ College of Information Engineering, Shaoyang University, Shaoyang, 422000 Hunan, China \\ ${ }^{2}$ Institute of Data Science, City University of Macau, Macau, 999078 Macau, China \\ ${ }^{3}$ State Key Laboratory of Marine Resource Utilization in South China Sea, Hainan University, Haikou, 570228 Hainan, China \\ ${ }^{4}$ College of Physical Science and Engineering, Yichun University, Yichun, 336000 Jiangxi, China
}

Correspondence should be addressed to Han Wang; hanwang1214@126.com and Liangpeng Lu; d18092100036@cityu.mo

Received 20 January 2021; Revised 24 February 2021; Accepted 28 March 2021; Published 3 May 2021

Academic Editor: Jian Feng Li

Copyright (c) 2021 Liang Zong et al. This is an open access article distributed under the Creative Commons Attribution License, which permits unrestricted use, distribution, and reproduction in any medium, provided the original work is properly cited.

In direct position determination (DPD), a large number of observation data need to be integrated and transmitted, which creates higher requirements for the transmission performance of the network. To alleviate the problem of performance degradation in a large number of data transmissions, this paper proposes a heterogeneous network architecture and transmission control algorithm for cross-regional heterogeneous networks. Through the heterogeneous integration of satellite and multihop networks, a transmission control model suitable for the long delay and high bit error rate environments is established, the congestion window of each stage of network transmission is analyzed, and the efficiency and accuracy of the algorithm are verified by experiments. The results show that a large amount of data can be transmitted in a heterogeneous network. When dealing with direct location, the algorithm can effectively transmit a large number of observation data for cross-regional heterogeneous networks. This simple and applicable transmission control algorithm can improve the satellite link throughput and reduce the download response time compared with the traditional transmission algorithm. These studies provide a reference for a large number of data transmissions in direct position determination.

\section{Introduction}

Positioning technology plays an important role in the fields of wireless signal processing, sensor monitoring, satellite navigation, and target detection [1-3]. Especially in the application of passive positioning, the common applications are passive positioning of radar, communication systems, and positioning of low SNR aircraft. In the civil field, the most common is the various monitoring and real-time positioning functions of wireless sensors [4-6].

The performance of DPD depends on obtaining the location and speed information of the transmitter or passive target from the original data received by the receiving station, which has a large number of data transmissions and easily causes network congestion. In different scenarios, the final solution of DPD is achieved by multidimensional grid search. However, grid search creates many computations. The transmission of raw data to the fusion center will occupy a large bandwidth, which will further increase the network transmission load and degrade its performance.

With the popularization and application of mobile Internet and Internet of Things (IoT), people increasingly focus on cross-regional positioning technology. To improve the transmission performance of a large quantity of observation data in cross-regional networks, researchers have conducted numerous studies [7-9], but further research is needed to improve the performance of network data transmission under long delays and high bit error rates (BERs). Based on this, researchers have conducted research on the transmission performance in the heterogeneous environment of wired 
and wireless networks [10-12]. However, there is still a lack of effective solutions for research on how to effectively enhance the transmission of heterogeneous satellite and terrestrial multihop networks. Therefore, it is an urgent problem to establish an efficient and simple algorithm to adapt to heterogeneous network environments with long delays and high bit error rates.

This paper proposes a transmission control algorithm for heterogeneous networks that is suitable for crossregion DPD. A transmission control mechanism suitable for a long delay and high bit error rate environment is established, and the congestion windows in each stage of network transmission are analyzed. This mechanism provides a feasible scheme for a large number of data fusion transmissions in DPD.

\section{State of the Art}

At present, researchers have performed considerable work on transmission performance under heterogeneous wireless network environments. Barakat et al. [13] conducted extensive research on the application of TCP to heterogeneous networks. The long delay, high bit error rate, and bandwidth asymmetry characteristics of heterogeneous networks were all important factors that caused TCP performance degradation. In addition, this paper also considered the possible impact of different network paths on the transmission performance and noted that future improvement schemes can enable TCP to be applied in end-toend heterogeneous networks. However, the study did not give a specific implementation to solve the problem of heterogeneous network transmission.

Oliveira et al. [14] deeply discussed the heterogeneous integration of mobile ad hoc networks (MANETs) and satellite networks. From the current research trend, satellites can be an important part of MANET routing. Therefore, a hybrid MANET-satellite network will play an important role in improving cross-regional data transmission. The paper also introduced the optimization of network resources and link availability, the provision of quality of service (QoS) and quality of experience (QoE), and the minimization of cost and energy supply and pointed out that the end-to-end optimization scheme can solve the above problems. However, this paper did not give a specific end-to-end solution. Based on integrated MANET and satellite network architecture, Miao et al. [15] further discussed the integrated network and pointed out that unpredictable node mobility, lack of central coordination, and limited available resources were important factors restricting the network performance. To solve the above problems, the choice of a gateway as a solution to achieve load balancing may be a direction of future research. The paper did not propose a solution mechanism for how to select the gateway and the specific implementation.

To meet the needs of emergency communication, according to the respective advantages of MANET, mobile communication, and satellite networks, Miao et al. [16] designed MANET-satellite and MANET-cellular. This paper noted that the common characteristics of the two systems need to be addressed and their architectures distinguished, which can provide powerful help for future research on routing protocols, QoS, energy efficiency, privacy protection, and resource management. Similarly, the paper also discussed mobile data download, the smaller storage space of the terminal, and the energy supply of the mobile device. However, this paper did not give a specific solution on how to realize the effective transmission mechanism of the two integrated systems.

To provide reliable wireless communication in postdisaster scenes, Yang et al. [17] proposed a hybrid network solution through terrestrial MANET and satellite networks. Compared with the conventional AODV (ad hoc ondemand distance vector) and AOMDV (ad hoc on-demand multipath) routing protocols, this solution can achieve a higher packet transmission rate, higher throughput, and lower delay. In addition, it can balance the traffic load of the gateway and maximize the use of satellite links. Under the above advantages, the hybrid network can provide strong support for postdisaster rescue. However, this paper did not give a specific routing protocol for the hybrid network. Considering that LEO satellite networks can provide large capacity and low delay network coverage and that their low orbit characteristics can reduce the size of ground terminals and enhance the mobility of ground networks, Yang et al.[18] provided an integrated network composed of a newgeneration LEO satellite network and ground wireless ad hoc network. The corresponding software simulation tool was adopted for different network hierarchies, which played a reference role for further research on satellite network architecture and ad hoc technology. However, the paper did not give a specific implementation mechanism. Considering the autonomy and flexibility of satellites and MANETs, Xie et al. [19] constructed the basic model of a hybrid MANETsatellite network and discussed the performance and applicability of ad hoc routing protocols in the network. Furthermore, it was noted that the self-organizing routing protocol can provide better performance in the above network architecture. However, this paper only simulated the existing MANET routing and did not give a routing protocol suitable for hybrid MANET-satellite networks.

Wei [20] gave the composition, network form, and main characteristics of the air-space-ground integrated information network and summarized the development status and trend of the integrated information network. It also analyzed the key technologies that need to be broken through to build the integrated information network. Research on MANETs has made remarkable progress in recent years. Chaudhary [21] used MANETs in combination with satellite networks for emergency situations caused by natural disasters or military conflicts. This paper reviewed the existing work on the integration of MANETs with satellite networks and the problems related to integration with satellite systems.

Zhu et al. [22] studied the TCP enhancement performance of a hybrid terrestrial satellite network. A comparative study of propagation delay, data segment loss rate, and buffer size was performed by two schemes, TCP splitting and endto-end E2E TCP. However, this paper did not propose a specific and effective heterogeneous network transmission 
scheme. In the past few years, space-air-ground integrated networks (SAGINs) have aroused extensive research interest. Liu et al. [23] reviewed the latest research work on SAGIN, from network design and resource allocation to performance analysis and optimization. After discussing several existing network architectures, this paper also pointed out some technical challenges and future directions.

Considering the considerable quantity of data brought by multinetwork search in DPD, Du et al. [24] proposed a novel geolocation architecture, which used a relatively simple channel assumption and a simplified cost function of multiple signal classification (MUSIC) algorithm to avoid highdimensional search. Du et al. [25] studied the cost function and performance of the DPD method in the presence of multipath propagation. In the case of multipath propagation, a general DPD model was established, and eight cost functions were developed to reduce the data transmission.

The demand for satellite navigation is growing exponentially. Due to technological advances, even handheld devices (mobile phones) can perform location-based services. Bhardwaj et al. [26] provided a brief review of satellite navigation, such as the working principle, position determination method, type of reference system for navigation applications, and satellite measurement technology. Kur et al. [27] studied the intersatellite link (ISL) to improve the accuracy of orbit determination and satellite clock estimation. The preparatory evaluation for the development of seven connectivity schemes for the precise orbit determination of three types of constellations showed that the clock estimation errors of satellites and stations were almost equal for all the connectivity schemes used. The clock result was not affected by ISL deviation. This study showed that ISL technology was a promising supplement to next-generation satellite navigation systems, and it can be recommended to use sequential connection and ring connection schemes for future navigation constellations.

The above research results mainly focus on the performance of heterogeneous networks, especially in the respective solutions of terrestrial MANETs or satellite networks. However, there are few studies on the transmission control of the hybrid network of terrestrial MANETs and satellite networks. This paper proposes a heterogeneous network architecture and transmission control algorithm for crossregional DPD. In this paper, a heterogeneous network transmission control model is established, and the deterministic analysis of the congestion window in a heterogeneous network transmission model is carried out. By analyzing the congestion window changes in slow start and congestion avoidance, an effective and reliable transmission mechanism is proposed. It provides a reference for heterogeneous network transmission suitable for cross-regional DPD.

The rest of this paper is organized as follows. In the third section, the solution of the hybrid network of terrestrial MANETs and satellite networks is proposed, and the congestion window of the slow start and congestion avoidance phases is determined. The fourth section analyzes the performance characteristics of the scheme through simulation and obtains the feasibility and efficiency of the pro- posed scheme. The last section summarizes this paper and gives relevant conclusions.

\section{Heterogeneous Network Architecture and Transmission Scheme}

In this paper, we propose a new heterogeneous network architecture and transmission control algorithm, which is aimed at efficiently transmitting a large quantity of data generated by cross-regional DPD. First, a heterogeneous network architecture suitable for cross-regional DPD is constructed, and then a new transmission mechanism is proposed, which uses the characteristics of the large bandwidthdelay product in heterogeneous networks to send large quantities of data to make full use of the links in heterogeneous networks. Finally, the data backlog value is used to distinguish the data loss caused by a large quantity of data generated during DPD to improve the performance of heterogeneous network transmission performance.

3.1. Network Architecture of Cross-Regional DPD. Positioning technology has been widely used in wireless multihop networks, and its data transmission depends on the performance of multihop networks. In DPD, the quantity of data generated by terminal equipment is large, so a more efficient and practical network is needed for cross-regional transmission. This paper constructs a heterogeneous network architecture that integrates a satellite network and a multihop emitter network constructed by terminal equipment. Multihop networks can be deployed quickly and conveniently without the support of infrastructure. The satellite network has the characteristics of wide coverage across regions. The heterogeneity of the two allows the scope of communication to be further expanded, as shown in Figure 1.

Passive location is a kind of location system in which the receiving station does not transmit signals but only completes the target location based on the observation signal. Generally, emitter location systems can be divided into emitter location systems based on the target's signal and external emitter location systems based on thirdparty emitters. The emitter location uses the signal emitted by the target to locate, while the external emitter location is realized by receiving the reflected signal from the thirdparty illuminator and the direct wave signal from the illuminator. In the DPD, multiarray observation points are used to collect a large quantity of data. In the heterogeneous network, the nodes in the multihop network can be observation points, and cross-regional positioning data transmission is realized through the satellite network.

The PDP needs a multidimensional grid search to achieve location, so it has high complexity and a long running time. For various DPD algorithms in different scenarios, the final solution is achieved by multidimensional grid search. The following problem is the large number of computations created by grid search. Moreover, the transmission of raw data to the fusion center takes up considerable bandwidth. The heterogeneous network architecture can not only achieve cross-data transmission but also improve the 


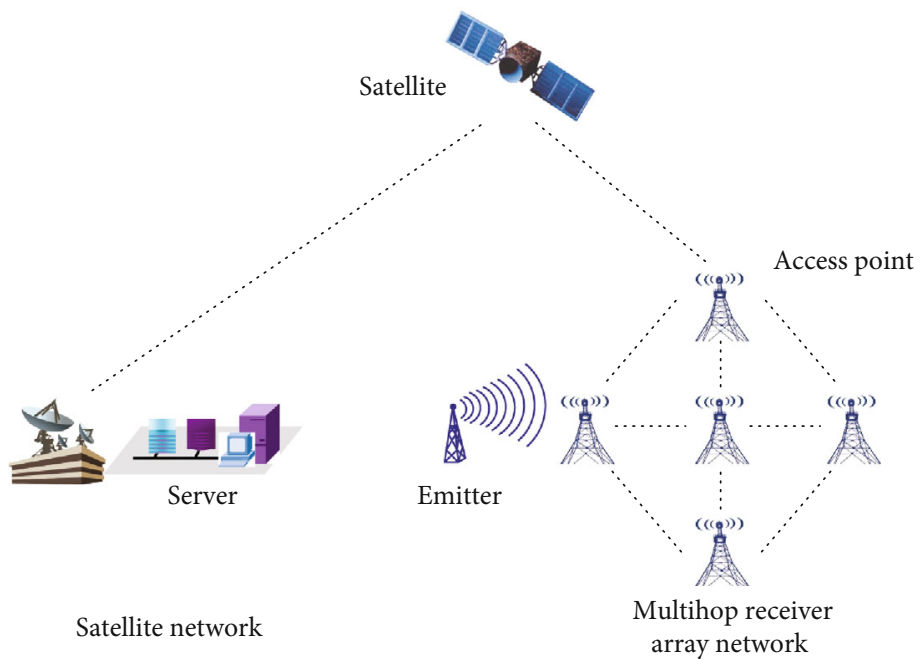

FIGURE 1: Heterogeneous network architecture of cross-regional direct location.

network performance when a large quantity of data is transmitted in a short time.

3.2. Slow Start. To apply the transmission mechanism of the integrated satellite and multihop network constructed above, it is necessary to increase the quantity of data in the slow start to meet the high bandwidth-delay product of the satellite network. First, a dynamic factor $\delta$ is introduced:

$$
\delta=\frac{\mathrm{RTT}}{\alpha}
$$

To normalize the round trip time, $\alpha$ is set as the reference time. Because round trip time can feedback the transmission state of the network in time, the congestion window of a slow start is adjusted by a dynamic factor. The dynamic factor changes according to the change in round trip time, which can determine the time consumed by data transmission in the network, which is an important basis for whether network congestion occurs. It depends on the data round trip time. Then, the congestion window is set to two different values to suit different network transmission environments.

The initial congestion window value can be set to SMSS $* \sqrt{\delta} / 4$. The SMSS is the maximum segment size set by the sender. Considering the different network states of the integrated network, the slow start is divided into two different intervals, corresponding to two different congestion windows. The first congestion window value is set to cwnd $+=$ $\mathrm{SMSS} * \sqrt{\delta} / 4$.

At this time, the congestion window is small, corresponding to the initial stage of slow start, to prevent the conflict caused by the transmission of other data in the integrated network. In addition, when the network state is in the saturation stage, such a small congestion window can also be set to avoid data congestion in the integrated network. The second congestion window value is set to cwnd $+=\operatorname{SMSS} * \sqrt{\delta}$.

At this time, the congestion window value is larger, corresponding to the situation in which the network state is better in the slow start, and a large quantity of data can be transmitted at this time.

Different DPD algorithms in various scenarios generate a large quantity of data through a multidimensional grid search. The transmission of these raw data to the integrated satellite and multihop network will take up considerable bandwidth. The above slow start strategy is aimed at alleviating the congestion and inefficiency caused by a large number of data transmissions. Through the judgment of different network conditions, the data transmission in the integrated network can be predicted, and the value of the congestion window can be set reasonably.

3.3. Distinction of Data Loss. In the DPD, target information is fused into the data stream collected by multiple observation stations, which leads to the characteristics of data accumulation and burst. These characteristics make the data transmission in heterogeneous networks prone to congestion loss. In the integrated network of satellites and multihop networks, the environment of wireless transmission will inevitably lead to the random loss of data.

Congestion loss and random loss of data are the main reasons for the performance degradation of integrated networks. The proposed algorithm not only improves the data transmission in the slow start but also detects the data loss in congestion avoidance.

The algorithm defines the data backlog value $\omega$, which is the unconfirmed data value in the integrated network.

$$
\omega=\mathrm{v} * t
$$

where $\mathrm{v}$ is the data transmission rate, which is the ratio of the transmission congestion window to the round trip time and $t$ is the difference between the round trip time and minimum round trip time.

$$
\begin{aligned}
& v=\frac{\mathrm{CWND}}{\mathrm{RTT}}, \\
& t=\mathrm{RTT}^{-\mathrm{RTT}_{\text {min }} .}
\end{aligned}
$$


Substituting formulas (3) and (4) into formula (2), then

$$
\begin{aligned}
& \omega=\frac{\mathrm{CWND}}{\mathrm{RTT}} *\left(\mathrm{RTT}_{-} \mathrm{RTT}_{\text {min }}\right), \\
& \omega=\mathrm{CWND} *\left(1-\frac{\mathrm{RTT}_{\text {min }}}{\mathrm{RTT}}\right) .
\end{aligned}
$$

A round trip time variation factor $\theta$ is introduced, which is the ratio of the minimum round trip time to the actual round trip time. The above formula is

$$
\omega=\mathrm{CWND} *(1-\theta) .
$$

The round trip time variation factor $\theta$ is a good reflection of the quantity of unconfirmed data in the network. When the round trip time is close to the minimum round trip time, then the network condition is better, the backlog value is smaller, and the number of data transmissions can be increased. In contrast, with the increase in round trip time, the backlog value increases, and there is a large quantity of unconfirmed data in the network, so the integrated network is prone to congestion, which reduces the number of data transmissions.

3.4. Congestion Avoidance. According to the data backlog value $\omega$, the transmission status of data in the integrated network can be well predicted so that the congestion loss or random loss of data can be judged. Therefore, in the congestion avoidance phase, the proposed algorithm adjusts the threshold appropriately to adapt to different network conditions.

The data backlog value of the $i$ th round trip time is defined as $\omega(\mathrm{i})$. To more accurately reflect the data backlog value in the network, the mean value of the three backlog values $[i-2, i]$ is finally taken. The mean vale $\omega_{\mathrm{AVG}}(\mathrm{i})$ is defined as follows:

$$
\omega_{\mathrm{AVG}}(\mathrm{i})=\frac{1}{3} * \sum_{k=i-2}^{i} \omega(k)
$$

Due to the wireless transmission environment of heterogeneous networks, the data backlog value may fluctuate violently, and the average value combined with the past two moments and the present data backlog value can more accurately reflect the network situation. The congestion avoidance phase of the proposed scheme is divided into three cases. In the first case, if $\omega_{\mathrm{AVG}}(\mathrm{i})$ is less than the boundary value $\omega_{1}$, it can be judged that the network condition is good, and the threshold value can be increased appropriately. At this time, the threshold is set to $4 * \mathrm{cwnd} / 5$. In the second case, when $\omega_{\mathrm{AVG}}(\mathrm{i})$ is not less than the boundary value $\omega_{1}$ and less than the boundary value $\omega_{2}$, it can be judged that the network condition is moderate and the threshold value can be adjusted appropriately. The threshold is set to $3 *$ cwnd/5. In the last case, if $\omega_{\mathrm{AVG}}(\mathrm{i})$ is not less than the boundary value $\omega_{2}$, it can be judged that the network condition is poor and the threshold value can be appropriately reduced. At this time, the threshold is set to $2 *$ cwnd $/ 5$.

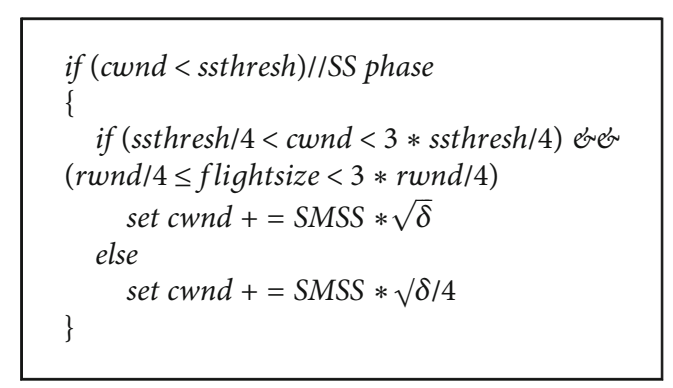

Algorithm 1

$\omega_{1}$ and $\omega_{2}$ are the boundary values to be adjusted and $\omega_{2}$ is greater than $\omega_{1}$. The proposed algorithm not only improves the transmission of initial DPD data but also detects the loss of data in a wireless transmission environment to reasonably adjust the threshold.

\section{Result Analysis and Discussion}

In the heterogeneous network, the nodes in the multihop network can be set as observation points. In the DPD, multiple observation points are used to collect a large quantity of data, and cross-regional data are transmitted through the satellite network. In the simulation topology, multihop nodes are used to simulate the observation nodes in the integrated network, and the multihop nodes are similar to the observation nodes in DPD. After the multihop nodes hop node by node, they connect to the satellite network through the access point (1 hop point), and the satellite network finally connects to the ground wired network. The topology can transmit a large quantity of data generated by DPD, as shown in Figure 2.

The transmission bandwidth is $1.54 \mathrm{Mbps}$ in the satellite network, and the one-way propagation delay is assumed to be the geostationary orbit satellite delay, which is set to $250 \mathrm{~ms}$. The simulation tests the bit error rate of the satellite link at $10^{-9}$ and $10^{-5}$.

The key part of the integrated network is the satellite network. Considering the high cost of satellite networks, the transmission performance of satellite networks must be improved, and the throughput of satellite links is the key parameter. To test the throughput of the satellite link in the integrated network and consider the number of nodes in the multihop network, the throughput of different nodes and satellite networks is tested under different bit error rates, as shown in the figure below.

Figure 3 shows that when the BER is $10^{-9}$, the three algorithms are simulated on different multihop nodes, and the throughput of the proposed scheme is greatly improved compared with TCP Reno and TCP Veno. TCP Reno is a classic transmission control protocol in wired networks. Because the bit error rate of the wired network is very low, the probability of random data loss is very low. However, in multihop networks and satellite networks, wireless transmission will lead to random loss of a large quantity of data, thus reducing the overall performance of the wireless network. It can be seen in the figure that in the test of the 1-hop node to the 5 -hop node, the performance of TCP Reno is maintained at approximately $22 \mathrm{kbps}$ for different multihop nodes. 


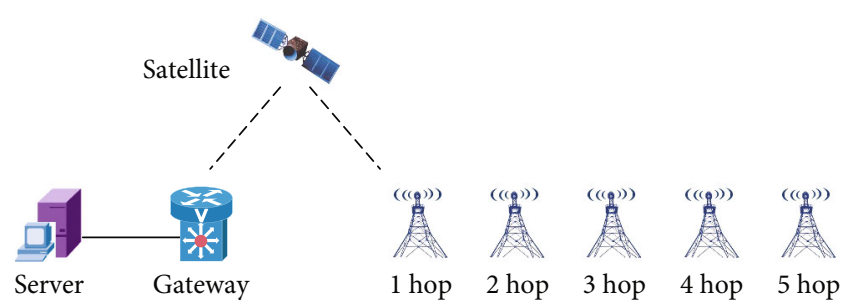

FIgURE 2: Simulation topology.

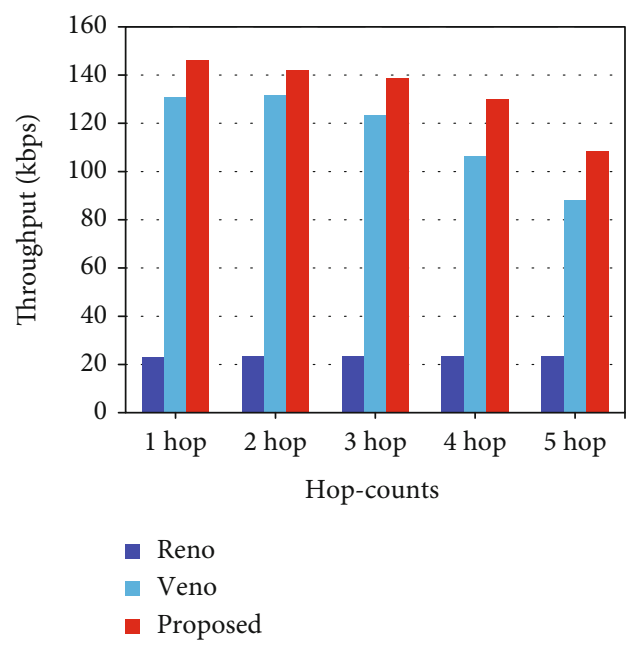

Figure 3: Throughput of satellite link when BER is $10^{-9}$.

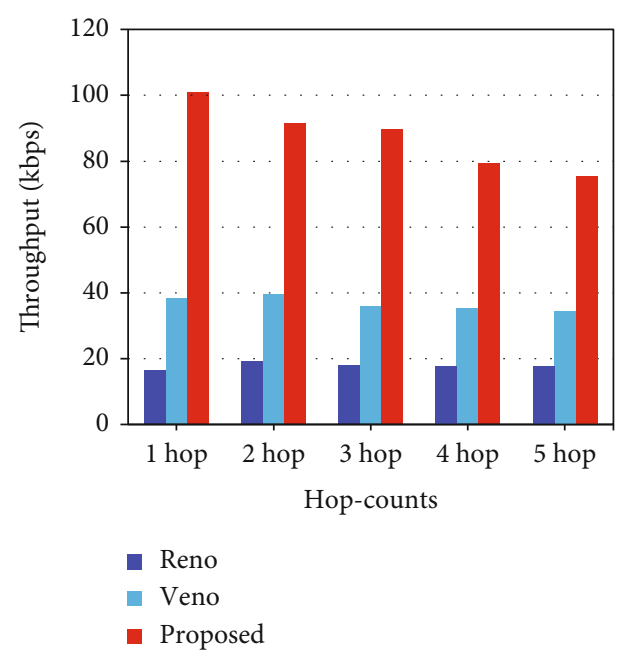

FIGURE 4: Throughput of satellite link when BER is $10^{-5}$.

However, the throughput of the other two algorithms decreases gradually.

TCP Veno is a transmission algorithm that uses the value of the data backlog in the network to judge the data loss. The algorithm is suitable for data loss in wireless communication. TCP Veno detects random data loss and adjusts the threshold of congestion avoidance according to different kinds of data loss, which improves the transmission performance in wireless networks. It can be seen in the figure that compared with increasing the volume of data transmission in the slow

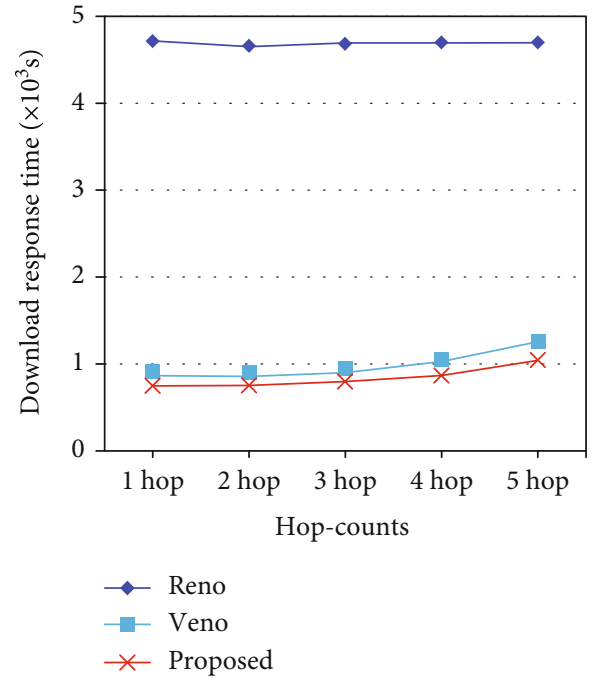

FIgURE 5: Download response time of nodes with BER of $10^{-9}$.

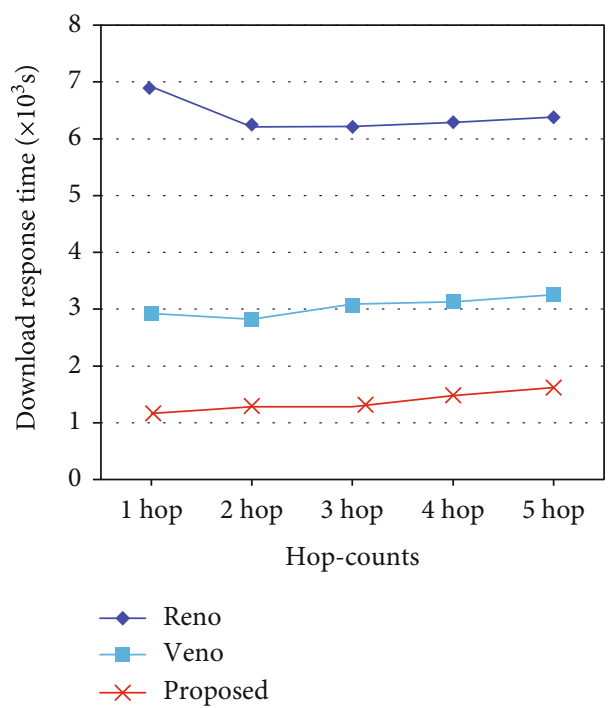

Figure 6: Download response time of nodes with BER of $10^{-5}$.

start, distinguishing the loss of different kinds of data can greatly improve the transmission performance of the integrated network. At 1-hop and 2-hop, the satellite throughput of TCP Veno is maintained at $130 \mathrm{kbps}$, which is approximately 6 times higher than that of TCP Reno.

The proposed algorithm not only distinguishes data loss but also improves the quantity of data injected into the network in the slow start to improve the transmission performance of the integrated network. The simulation results show that the throughput is $150 \mathrm{kbps}$ at the 1-hop node, which is approximately 7 times higher than TCP Reno. Compared with TCP Veno, the throughput is improved by $15.38 \%$. In the case of 5 -hop nodes, the throughput of TCP Reno is still maintained at approximately $22 \mathrm{kbps}$, and the throughput of TCP Veno is reduced to $90 \mathrm{kbps}$. The throughput of the proposed algorithm is $110 \mathrm{kbps}$, which is five times higher than that of TCP Reno and 22.22\% higher than TCP Veno. 
Figure 4 shows that when the BER is $10^{-5}$, the three algorithms are simulated on different multihop nodes, and the throughput of the proposed scheme is greatly improved compared with TCP Reno and TCP Veno. As seen in the figure, in the test of the 1-hop node to the 5-hop node, the throughput of TCP Reno is maintained at approximately $20 \mathrm{kbps}$ for different multihop nodes. The throughput of TCP Veno is approximately $40 \mathrm{kbps}$. At the 1-hop node, the throughput of the proposed algorithm is $100 \mathrm{kbps}$, which is 5 times and 1.5 times higher than that of TCP Reno and TCP Veno, respectively. Compared with Figure 3 of BER is 10-9, in the case of a high bit error rate, the random loss of data is more serious. If the loss of this kind of data can be correctly identified, the threshold can be appropriately adjusted in congestion avoidance to adapt to the situation of the network. The throughput of TCP Reno is still $20 \mathrm{kbps}$, while that of TCP Veno is $37 \mathrm{kbps}$. The throughput of the proposed algorithm is $78 \mathrm{kbps}$, which is 3.9 and 1.37 times higher than that of TCP Reno and TCP Veno, respectively.

Figure 5 shows the download response time of the three algorithms at different hop nodes when the BER is $10^{-9}$. In congestion avoidance, TCP Reno considers that all data loss is caused by congestion. The threshold is set to half of the congestion window, which directly reduces the transmission performance of the integrated network. Therefore, the download response time of TCP Reno in the satellite link is maintained at $4.8 \times 10^{3} \mathrm{~s}$. The download response time of the proposed algorithm and TCP Veno increases with the number of hops. As seen in the figure, the download response time of TCPVeno is approximately $1.0 \times 10^{3} \mathrm{~s}$. The download response time of the proposed algorithm is between $0.8 \times 10^{3}$ $\mathrm{s}$ and $1.0 \times 10^{3} \mathrm{~s}$. It can be seen that the proposed algorithm can increase the quantity of data sent and correctly identify the random data loss, which is conducive to the correct adjustment of the threshold according to the network state in congestion avoidance. This reduces the download response time of the integrated network.

Figure 6 shows the download response time of the three algorithms at different hop nodes when the BER is $10^{-5}$. Compared with $10^{-9}$, the download response time of all algorithms increases with the number of hops, except that TCP Reno decreases from a 1-hop node to a 2-hop node. Compared with TCP Reno and TCP Veno, the proposed algorithm has more obvious advantages. In particular, compared with TCP Veno, the proposed algorithm reduces the response time by approximately 2 times.

\section{Conclusion}

To improve the performance of the transmission control algorithm in heterogeneous networks suitable for crossregional DPD, this paper proposes a new algorithm that improves the throughput of integrated networks and reduces the response time of multihop networks by increasing the quantity of data injected into satellite networks and distinguishing different kinds of data loss in wireless networks. The simulation experiment compares TCP Reno, TCP Veno, and the proposed algorithm. The results are as follows:
(1) In the integrated network, the performance of the proposed scheme is better than that of the other two transmission control algorithms

(2) In the case of data loss caused by the bit error rate, especially in the case of a high bit error rate, the performance of the proposed scheme can be improved more obviously

(3) In the slow start, the throughput of the satellite network can be improved by increasing the amount of data injection

In this study, a new optimization scheme is proposed by combining the experimental and theoretical research of heterogeneous networks, which is suitable for cross-regional positioning. Through the analysis of the integrated network, the size of the congestion window is modified in the slow start, and the size of the threshold is adjusted in the congestion avoidance. The established transmission mechanism can improve the transmission performance of heterogeneous networks, and follow-up research on the transmission control of heterogeneous networks with cross-regional DPD is carried out. The research has certain reference significance.

\section{Data Availability}

The data used to support the findings of this study are available from the corresponding author upon request.

\section{Conflicts of Interest}

The authors declare that there are no conflicts of interest regarding the publication of this paper.

\section{Acknowledgments}

This work was supported by the Hunan Provincial Natural Science Foundation (Grant No. 2020JJ4557), the Scientific Research Fund of Hunan Provincial Education Department (Grant Nos. 20B519, 19B512, and 19A446), the Open Project of State Key Laboratory of Marine Resources Utilization in South China Sea (Grant No. MRUKF2021034), the Project of the National Natural Science Foundation of China (Grant No. 61961014), and the Jiangxi Provincial Natural Science Foundation (Grant No. 20202BABL212001).

\section{References}

[1] F. Ma, Z. M. Liu, and F. Guo, "Distributed direct position determination," IEEE Transactions on Vehicular Technology, vol. 69, no. 11, pp. 14007-14012, 2020.

[2] J. Li, P. Ma, X. Zhang, and G. Zhao, "Improved DFT algorithm for 2D DOA estimation based on 1D nested array motion," IEEE Communications Letters, vol. 24, no. 9, pp. 1953-1956, 2020.

[3] J. Li, Y. He, P. Ma, X. Zhang, and Q. Wu, "Direction of arrival estimation using sparse nested arrays with coprime displacement," IEEE Sensors Journal, vol. 21, no. 4, pp. 5282-5291, 2021. 
[4] X. Li, M. Zhao, Y. Liu, L. Li, Z. Ding, and A. Nallanathan, "Secrecy analysis of ambient backscatter NOMA systems under I/Q imbalance," IEEE Transactions on Vehicular Technology, vol. 69, no. 10, pp. 12286-12290, 2020.

[5] A. J. Weiss, "Direct position determination of narrowband radio frequency transmitters," IEEE Signal Processing Letters, vol. 11, no. 5, pp. 513-516, 2004.

[6] H. Wang, L. Xu, Z. Yan, and T. A. Gulliver, "Low-complexity MIMO-FBMC sparse channel parameter estimation for industrial big data communications," IEEE Transactions on Industrial Informatics, vol. 17, no. 5, pp. 3422-3430, 2021.

[7] X. Wang, L. Wan, M. Huang, C. Shen, Z. Han, and T. Zhu, "Low-complexity channel estimation for circular and noncircular signals in virtual MIMO vehicle communication systems," IEEE Transactions on Vehicular Technology, vol. 69, no. 4, pp. 3916-3928, 2020.

[8] D. Meng, X. Wang, M. Huang, L. Wan, and B. Zhang, "Robust weighted subspace fitting for DOA estimation via block sparse recovery," IEEE Communications Letters, vol. 24, no. 3, pp. 563-567, 2020.

[9] X. Li, Q. Wang, Y. Liu, T. A. Tsiftsis, Z. Ding, and A. Nallanathan, "UAV-Aided multi-way NOMA networks with residual hardware impairments," IEEE Wireless Communications Letters, vol. 9, no. 9, pp. 1538-1542, 2020.

[10] W. Wen, Y. Cui, F. C. Zheng, S. Jin, and Y. Jiang, "Random caching based cooperative transmission in heterogeneous wireless networks," IEEE Transactions on Communications, vol. 66, no. 7, pp. 2809-2825, 2018.

[11] X. Wang, M. Huang, and L. Wan, "Joint 2D-DOD and 2DDOA estimation for coprime EMVS-MIMO radar," Circuits, Systems, and Signal Processing, 2021.

[12] N. Raveendran, H. Zhang, D. Niyato, F. Yang, J. Song, and Z. Han, "VLC and D2D heterogeneous network optimization: a reinforcement learning approach based on equilibrium problems with equilibrium constraints," IEEE Transactions on Wireless Communications, vol. 18, no. 2, pp. 1115-1127, 2019.

[13] C. Barakat, E. Altman, and W. Dabbous, "On TCP performance in a heterogeneous network: a survey," IEEE Communications Magazine, vol. 38, no. 1, pp. 40-46, 2000.

[14] A. Oliveira, Z. Sun, M. Monier et al., "On optimizing hybrid ad-hoc and satellite networks-the MONET approach," in 2010 Future Network \& Mobile Summit, pp. 1-8, Florence, Italy, June 2010.

[15] Y. Miao, Z. Sun, F. Yao, N. Wang, and H. S. Cruickshank, "Study on research challenges and optimization for internetworking of hybrid MANET and satellite networks," in Personal Satellite Services. PSATS 2013. Lecture Notes of the Institute for Computer Sciences, Social Informatics and Telecommunications Engineering, vol 123, R. Dhaou, A. L. Beylot, M. J. Montpetit, D. Lucani, and L. Mucchi, Eds., pp. 90-101, Springer, Cham, Toulouse, France, 2013.

[16] Y. Miao, Z. Sun, N. Wang, and H. Cruickshank, "Comparison studies of MANET-satellite and MANET-cellular networks integrations," in 2015 International Conference on Wireless Communications \& Signal Processing (WCSP), pp. 1-5, Nanjing, China, October 2015.

[17] X. Yang, Z. Sun, Y. Miao, and H. Cruickshank, "QoS routing for MANET and satellite hybrid network to support disaster relives and management," in 2016 IEEE 83rd Vehicular Technology Conference (VTC Spring), pp. 1-5, Nanjing, China, May 2016.
[18] X. Yang, Z. L. Sun, H. F. Liu, K. Zhao, Z. J. Cheng, and Y. Miao, "Technology of new Generation LEO satellite networks and terrestrial MANET integration," ZTE Technology Journal, vol. 22, no. 4, pp. 58-63, 2016.

[19] X. Xie, J. Wang, X. Guo, and X. Wu, "Performance evaluation of ad-hoc routing protocols in hybrid MANET-satellite network," in Machine Learning and Intelligent Communications. MLICOM 2018. Lecture Notes of the Institute for Computer Sciences, Social Informatics and Telecommunications Engineering, vol 251, L. Meng and Y. Zhang, Eds., pp. 500-509, Springer, Cham, Hangzhou, China, 2018.

[20] W. Wu, "Survey on the development of space-integratedground information network," Space-Integrated-Ground Information Networks, vol. 1, no. 1, pp. 1-16, 2020.

[21] N. K. Chaudhary, "MANET-satellite network interoperability issues and challenges: an overview," International Journal of Trend in Research and Development, vol. 3, no. 3, pp. 537539, 2016.

[22] J. Zhu, S. Roy, and J. H. Kim, "Performance modelling of TCP enhancements in terrestrial-satellite hybrid networks," IEEE/ACM Transactions on Networking, vol. 14, no. 4, pp. 753-766, 2006.

[23] J. Liu, Y. Shi, Z. M. Fadlullah, and N. Kato, "Space-air-ground integrated network: a survey," IEEE Communications Surveys \& Tutorials, vol. 20, no. 4, pp. 2714-2741, 2018.

[24] J. Du, D. Wang, W. Yu, and H. Yu, "Direct position determination of unknown signals in the presence of multipath propagation," Sensors, vol. 18, no. 3, p. 892, 2018.

[25] J. Du, H. Yu, D. Wang, D. Zhang, and G. Liu, "Unified subspace fitting framework and its performance analysis for direct position determination in the presence of multipath propagation," IEEE Access, vol. 7, pp. 6889-6914, 2019.

[26] S. C. Bhardwaj, S. Shekhar, A. Vidyarthi, and R. Prakash, "Satellite navigation and sources of errors in positioning: a review," in 2020 International Conference on Advances in Computing, Communication \& Materials (ICACCM), pp. 43-50, Dehradun, India, August 2020.

[27] T. Kur, T. Liwosz, and M. Kalarus, "The application of intersatellite links connectivity schemes in various satellite navigation systems for orbit and clock corrections determination: simulation study," Acta Geodaetica et Geophysica, vol. 56, pp. 1-28, 2020. 